\title{
AFRONTAMIENTOS DE ENFERMERAS AL ASUMIR LA GERENCIA ESCOLAR: UNA MIRADA DESDE LA TEORÍA DE ROY
}

\author{
O afrontamento das enfermeiras ao assumirem a gerência escolar: um olhar a partir da \\ teoria de Roy \\ Confrontation of nurses when assuming the school management: a look from Roy's \\ adaptation theory
}

Mariana Olizbeth Salinas Alvirde

Danelia Gómez Torres ${ }^{2}$

Vianey Méndez Salazar ${ }^{3}$

\section{RESUMEN}

Esta investigación aborda la transición gerencial de las instituciones educativas de médicos a enfermeras con objetivo de delimitar mecanismos de afrontamiento y modos adaptativos al iniciar la gestión en el entorno académico. Se realizó estudio cualitativo de corte histórico-social sustentado por la teoría de adaptación de Roy. Los sujetos estudiados fueron las primeras profesoras que ocuparon la dirección de escuelas universitarias de enfermería, a través de entrevistas se recolectaron los datos. Los resultados revelaron que la incursión de enfermeras permitió abordar el proceso salud-enfermedad de manera distinta al acto médico, lo que significó generar mecanismos de afrontamiento para trabajar en la construcción de paradigmas que conformarán un pensamiento profesional y visión disciplinaria propia, a través de un plan de estudio solido. Concluímos que se renovaron los métodos de trabajo, creándose nuevos niveles académicos, especializaciones y titularidades en las materias en base a sacrificio y dedicación, iniciándose su autonomía profesional.

Palabras Clave: Enfermería. Educación. Historia. Adaptación. Gerencia.

Resumo

Esta pesquisa aborda a transição gerencial das instituiçðes educativas de médicos às enfermeiras, com o objetivo de delimitar mecanismos de afrontamento e modos adaptativos ao iniciar a gestão no âmbito acadêmico. Realizou-se um estudo qualitativo de abordagem histórico-social, orientado pela teoria de adaptação de Roy. Os sujeitos estudados foram as primeiras professoras que ocuparam a direção das escolas universitárias de enfermagem; os dados foram recoletados por meio de entrevistas. Os resultados revelaram que a incursão de enfermeiras permitiu abordar o processo saúde-doença de maneira distinta ao ato médico, o que significou gerar mecanismos de afrontamento para trabalhar na construção de paradigmas que conformarão um pensamento profissional e uma visão disciplinar própria, a partir de um plano de estudo sólido. Concluiu-se que se renovarão os métodos de trabalho com a criação dos novos níveis acadêmicos, especializaçðes e titularidades nas matérias, com base em sacrifício e dedicação, iniciando-se sua autonomia profissional.

Palavras-chave: Enfermagem. Educação. História. Adaptação. Gerência.
The research emerged from the necessity of learning the manager transition from doctors to nurses at nursing educational institutions. The purpose was to differentiate between the mechanisms of afrontation and modes of adaptation in the academic sphere managing process. The qualitative research in socio-historical context is oriented by the Theory of Adaptation by Roy. The participants were the first female professors who held leading posts in universities; the interview was a data collection instrument. The results revealed the fact that the incursion of nurses made it possible to treat the health - illness process in a way different from medical activity, which meant the generation of mechanisms of confrontation in order to work on the construction of paradigms that will conform a professional thinking and a proper disciplinary vision based on a solid plan of study, The conclusions was the creation and renovation of working methods to achieve new academic levels, specializations and they become the heath of the class with a based on sacrifice and dedication, starting the professional autonomy.

Keywords: Nursing. Education. History. Adaptation. Managing.

'Pasante de la Licenciatura de Enfermería. Realizando Servicio Social en la Facultad de Enfermería y Obstetricia de la Universidad Autónoma del Estado de México. Toluca - Estado de México - México. E- mail: mariana.olizbeth@hotmail.com.; ²Doctora en Enfermería. Coordinadora de Investigación de la Facultad de Enfermería y Obstetricia de la Universidad Autónoma del Estado de México. Miembro del Cuerpo Académico Ejercicio de Enfermería FEYo UAEM. Toluca - Estado de México- México. E- mail: gomezdanelia@usa.net; ${ }^{3}$ Doctora en Educación. Profesora de tiempo completo de la Facultad de Enfermería y Obstetricia de la Universidad Autónoma del Estado de México. Toluca- Estado de México- México. E- mail: vianneyms@usa.net. 


\section{INTRODUCCIÓN}

El presente artículo es un apartado del proyecto de investigación, titulado "Nivel de adaptación al rol de enfermera gerente en las instituciones educativas", el espacio cronológico que comprendió este estudio de investigación fueron los años 70s- 80s, y es creado por la necesidad de dar a conocer las modificaciones en la gerencia de las escuelas de enfermería universitarias del centro de la República Mexicana, a través de la transición de gerente- médico a gerente- enfermera, lo que llevo a conseguir cambios significativos en la formación profesional reflejándose tanto en los niveles educativos, como en el ámbito laboral.

Un antecedente relevante del estudio es que existía una estrecha dependencia en el espacio escolar hacia el área médica, por ser ahí donde se ocupaban los cargos directivos, limitando el crecimiento profesional de enfermería en ese ámbito. La reforma educativa emite una nueva ley federal de educación en México (1973), buscando una modernización en la enseñanza, con nuevos requerimientos sociales para promover la flexibilidad de la educación, así mismo, a partir de los años 70s los subsidios a las universidades crecieron y en muchas de éstas surgieron nuevas carreras acordes a la estructura profesional' . Esta reforma educativa crea una inquietud en las enfermeras por ocupar un espacio dentro de las instituciones educativas. De esta manera, tal percepción sirve como punto de partida a las enfermeras en México para comprender que en realidad adquirir conocimientos es responsabilidad individual. Ese interés de las profesionistas en cuanto a la formación académica, las impulsa a iniciar un proceso de transformación, que les permitió obtener un campo propio de acción donde incursionan como directoras de escuelas formadoras de personal de enfermería, siendo ellas las únicas en tomar decisiones respecto a la profesión.

Uno de los retos que tiene la profesión de enfermería es la producción y fortalecimiento de conocimientos propios que permitan fortalecer la autonomía de las enfermeras, mejorar la calidad de atención que prestan a la sociedad y promover su reconocimiento como disciplina profesional2. Considerando que la fundamentación científica es un paso imprescindible en una investigación, se retoma la teoría de adaptación de Roy para conocer los modos de adaptación y mecanismos de afrontamiento. Por lo anterior, debemos tomar en cuenta que la teoría ayuda a adquirir conocimientos que perfeccionan la práctica mediante la descripción, explicación, predicción y control de los fenómenos ${ }^{3}$, pues:

La gran importancia en un campo científico no es acumular conocimientos, más si utilizarlos como instrumento para indagary actuar sobre la realidad. Hay diferencia entre tener apenas el conocimiento acumulado y tener el conocimiento y saberlo utilizar, el primero apenas aliena, ya el segundo te vuelve ser humano y su tarea es muy rica ${ }^{4}$.
El desarrollo de la enfermería como profesión, disciplina y ciencia, ha exigido a sus profesionales a lo largo de la historia hacer evidente, por medio de esfuerzo y lucha, la preparación fundamental que en relación al conocimiento sustente el ejercicio profesional de la enfermería como práctica social.

\section{MODELO Y TEORIA DE ADAPTACIÓN}

Roy ${ }^{5}$ considera su modelo como un método valioso para realizar los puntos comunes e independientes que existen en las profesiones de enfermería y medicina. Igualmente opina que su modelo ayuda a fomentar el desarrollo teórico de los que lo aplican ya sea en la práctica o en la investigación, quienes a la par se apoyan a contrastar las teorías existentes e indagar en la construcción de otras nuevas.

Por otra parte Roy también considera al hombre un ser biopsicosocial, en relación constante con el entorno, el cual es cambiante, pues un hombre es un complejo sistema biológico que trata de adaptarse a los aspectos de la vida ${ }^{6}$, para esta investigación el caso de adaptación se aplica a un nuevo rol, que es el de gerente de las instituciones de educación superior.

En esta misma vertiente Roy en su teoría desarrolló el proceso de afrontamiento y adaptación, derivada de su modelo conceptual, con el propósito de explicar el subsistema cognitivo y de esta manera identificar las habilidades que utiliza el individuo para controlar el estrés y promover su adaptación, uno de los conceptos importantes considerados en su teoría, y aplicado a este estudio es el afrontamiento el cual es denominado como esfuerzos comportamentales y cognitivos que realiza la persona para atender las demandas del ambiente, que actúan como un todo para mantener sus procesos de integridad.

Roy establece los modos de adaptación y los mecanismos de afrontamiento, a su vez define a estos últimos como las formas innatas o adquiridas de respuestas ante los cambios del entorno8. Donde el nivel de adaptación de una persona es un punto que cambia constantemente y esta constituida por estímulos. A este nivel de adaptación lo representa como la condición de los procesos de vida ${ }^{5: 1}$, y que representa la contraparte de el afrontamiento con respuestas adaptativas corrientes. Congruentemente estos postulados permiten responder al cuestionamiento del estudio y determinar el nivel de adaptación de las enfermeras en el puesto gerencial en escuelas de enfermería.

La aplicación de esta teoría en la temática, se debe a que Roy:i ${ }^{8}$ sostiene que un objetivo de la enfermería consiste en promover la adaptación del hombre a cada uno de los modos adaptativos, distinguiendo también entre ciencia de enfermería y ciencia médica, reclamando la impartición de los conocimientos de estas dos disciplinas en cursos independientes.

\section{METODO}

Para explicar el fenómeno de transición que se vivió en la dirección de escuelas de enfermería y poder ser comprendido como un proceso evolutivo de la profesión, se crea esta 
investigación con un enfoque cualitativo que permitió acercarnos al conocimiento sobre la experiencia vivida por las primeras enfermeras dirigentes en las escuelas de enfermería. Es de corte histórico-social porque se determina en base al fenómeno estudiado, y siguiendo los tres pasos: Recolección de datos, Evaluación crítica de los datos, Presentación de hechos y las Conclusiones. Tal método comprende el estudio de los grupos, siendo para este caso el grupo social de enfermería. Fueron siete los actores sociales seleccionados para esta investigación de escuelas y facultades de enfermería universitarias de la zona centro de la república mexicana y que limitaran con el Estado de México, se eligieron las primeras directoras de las escuelas de enfermería, que posteriormente se incorporaron a las universidades y en el caso de las directoras fallecidas, se solicitó la participación de las profesoras que estuvieron en el proceso de transición, siendo esos los criterios de selección.

Para la recolección de datos, el instrumento utilizado fue una guía de entrevista estructurada, la cual contenía una ficha de identificación institucional y seis preguntas utilizadas para la parte de este estudio. El instrumento se desarrollo bajo cuatro grandes categorías denominadas roles, aquí se presenta el rol académico exclusivamente, tratando aspectos como la construcción de paradigmas propios de enfermería, reestructuración de planes y programas de estudio, liderazgo y mecanismos generados para adaptarse a la jerarquía gerencial y finalmente se planteo una pregunta abierta que posibilitó la obtención de información relevante. Para el procesamiento de datos se organizó la información recolectada, se capturó el testimonio de las entrevistadas y posterior a su lectura, se trabajó con la técnica colorimétrica que facilito la selección de las unidades de análisis más sobresalientes con la finalidad de obtener datos que nos dieran a conocer el precedente académico durante su gestión.

En la evaluación crítica fue necesario hacer la validez de datos para determinar la calidad y relevancia de la información, iniciando desde la elaboración de la guía de entrevista hasta la interpretación de los datos para poder analizar los comportamientos de la gestión de las directorasenfermeras, así mismo, de esta manera efectuar la presentación de hechos y discutidos a la luz de los conceptos de la teoría de adaptación para llegar a conclusiones.

El fundamento teórico está basado en la teoría de adaptación de Roy como eje conductor en la investigación la cual centra su metaparadigma de enfermería al hombre, salud, medio ambiente y enfermera, ya que están relacionados en un todo global. Whetsell ${ }^{9}$ sostiene que lo que determina la calidad de vida de una persona no son las circunstancias que ocasionan estrés, sino la forma como la persona reacciona ante éstas y su habilidad para adaptarse. El aspecto ético de la investigación, se cumplió con lo establecido en el Reglamento de la Ley General de Salud Mexicana en materia de investigación, siendo el Art. 13 y 14 los utilizados. Para la aplicación de la entrevista se dio a conocer a las entrevistadas el consentimiento libre informado por escrito. De la misma manera se cumplió con el
Art. 16 en lo referente a proteger la privacidad del sujeto de investigación. De esta manera se sustentó el desarrollo de la investigación promoviendo la participación activa de los sujetos.

\section{RESULTADOS Y DISCUSION}

La formación de las enfermeras en nuestro país es un tema que siempre ha preocupado y ocupado a las propias enfermeras, de acuerdo a Roy ${ }^{10}$, la enfermera interviene para cambiar el ambiente y promover la adaptación a través de la expansión de las habilidades adaptativas del individuo, de donde surgen las características de la enfermería con respecto a su saber y nivel académico adquirido a través del tiempo, situación que durante los años 70s fue diferente en relación con otros profesionistas del área de la salud, por deducción se considera que si las enfermeras logran una igualdad académica, tendrán consecuentemente un reconocimiento similar al de otros profesionistas. Precisamente desde los inicios de la profesión se ha tenido una evolución, tanto en la práctica como en los modelos de formación, tratando de dar con esto, respuesta a las necesidades de la población y a los propósitos y tendencias de los servicios de salud.

\section{Adaptacion de Enfermeria al proceso de transición}

En América Latina, las primeras escuelas de enfermería surgieron en las últimas décadas del siglo XX, generalmente bajo la dirección de profesionales de la medicina ${ }^{11}$. Las primeras características de la etapa del sistema educativo de enfermería en México corresponden a la transición de las escuelas de enfermería respecto a las de medicina, integrándose al sistema universitario de forma independiente. En esa década, crece la preocupación de las enfermeras por conseguir mayores niveles académicos, buscando sistematizar el conocimiento amplio y empírico que aumentara la concepción del proceso de atención de enfermería en pos de un diagnóstico preciso en enfermería, al abordar el proceso salud-enfermedad de manera distinta al acto médico, lo que significó generar mecanismos de afrontamiento y trabajar en la construcción de paradigmas que conformaran un pensamiento profesional propio, ampliando la manera de entenderse a sí misma y al objeto de su actividad profesional, al visualizarse las competencias para ejercer la gerencia se inicia esa fase de cambio, por lo que al indagar a las entrevistadas sobre este proceso, mencionaron:

Yo creo que veían como factible el hecho de que pudiera ser una enfermera directora, que ya era el momento, estábamos preparadas, habíamos dado ya muestra de que estábamos a la misma altura de participación (...) trate de que tuviera realmente los conocimientos y las habilidades, las competencias (...) como para hacer un papel digno al frente de la dirección (...) es asíque a partir de ahíya no regreso ningún médico a la dirección (E1). 
Tal condición se aplicó tanto al ámbito académico como al campo profesional; siendo este hecho el más significativo para la enfermería mexicana, en primera instancia conseguir que las escuelas de enfermería fueran dirigidas por enfermeras ${ }^{12}$. Todo este movimiento fue paralelo a las reformas sucedidas en la profesión y al lograr la aparición de direcciones académicas de enfermería se limito la participación de los médicos como dirigentes de las instituciones de educación, para designar a las propias profesionistas enfermeras.

La enfermería como disciplina, tenía un papel fundamental en la formación y orientación de su personal de acuerdo con los cambios que se generaban en aquel momento, debiendo ser congruentes con las necesidades de los servicios de salud. Es evidente que para desempeñar ciertas funciones, la enfermera requería mejorar su formación básica, dominar el arte de la enfermería cuyas bases se han sustentado en las ciencias biológicas y sociales y, debían estar especialmente capacitadas para la enseñanza y la administración ${ }^{13}$. Destacando con esto que la preparación del personal de enfermería, para ejercer funciones de mayor responsabilidad requería una orientación especial y un nivel relativamente alto de educación que le permitieran desarrollar los conocimientos y las aptitudes en un sentido más amplio, de esta forma, adaptarse a las necesidades que como dirigente se requerían para ejercer de manera eficaz las funciones acordes a las reformas educativas.

\section{Mecanismos de afrontamiento en planes y programas}

En la mayoría de escuelas y facultades de enfermería del país, durante el periodo -1970-1980, prevaleció básicamente el plan de estudios de nivel técnico, creando y reestructurando sus planes de estudio en base al enfoque teórico denominado tecnología educativa, por ello, la importancia de cuestionar a las entrevistadas en relación con la reestructuración de planes y programas de estudio en tal época, obteniendo que:

Fue la óptima de la tecnología educativa entonces todo el mundo trabajaba nuestros programas $(E-1)$

Se implanto un plan de estudios les innovador, fabuloso que al rato lo tenían todo México, hasta parte de Latinoamérica (...) lo adoptaron muchas escuelas, fue revolucionario (E-5).

Como se refleja, los retos a los que se enfrentaban las instituciones académicas de Enfermería en el periodo estudiado eran: evaluar los planes y programas educativos, en donde el eje fundamental de la enseñanza debía ser el cuidado del paciente e implementar cursos que favorecieran la educación permanente, así como vincular los programas educativos con la práctica.
En otro sentido los seres humanos se adaptan a través de procesos de aprendizaje adquiridos a través del tiempo; por esta razón, el contexto forma parte de un sistema del cual no se puede separar al individuo, pues actúa como sistema de amortiguación del estimulo estableciendo el nivel previo a la situación, y posterior del individuo (gerente). En congruencia con Roy ${ }^{10: 1}$ al mencionar que el ser humano con sus decisiones es responsable de la integración de procesos y comparten poder creativo, es decir, que la persona tiene la capacidad de decidir y de participar activamente en su proceso de adaptación; en efecto, las decisiones se deben tomar conjuntamente entre los involucrados. Al realizar cambios en los planes y programas de estudio en enfermería existe la opción de tomar una conciencia de la realidad y el desarrollo del juicio crítico, la iniciativa para actuar como agentes de cambio se encuentra en la educación que se imparte dentro de las instituciones, pues esta propicia el compromiso que como profesionista y persona corresponde desempeñar, ya que la educación tiene como fin transformar para contribuir al desarrollo humano.

Otro mecanismo de afrontamiento fue el cambio en la titularidad de asignaturas impartidas en un inicio por médicos, es decir la preparación de las estudiantes era controlada en su totalidad por la profesión médica, pero de algún modo se inició la enseñanza del conocimiento y el saber por parte de enfermería, lo cual le permitió a esta disciplina recobrar un rol que sería demostrado a través de capacidad y pericia. La formación y actualización fue uno de los ejes que guió a las nuevas enfermeras directoras por el camino del reconocimiento y prestigio, permitiendo con esto el aumento de enfermeras dentro de la planta docente, como se constata en la respuesta siguiente:

Teníamos que ir a la práctica con las alumnas y asíde acuerdo a las aptitudes y capacidades de cada una se iba asignando las materias (...) Se platico y acordó tanto con las colegas enfermeras como con los médicos que aun continuaron en la escuela que ellos dieran lo medico y la enfermera lo de enfermería. ( $E$ 6)

Es importante señalar que este mecanismo de afrontamiento se acompaña por otro dato significativo, una vez alcanzada la meta de poder ser responsables de asignaturas, quedaba por conseguir la titularidad, es decir; obtener plaza docente, con lo que las enfermeras consiguieron ser docentes únicas en asignaturas de enfermería, tanto en el aspecto teórico como en el práctico así como de las evaluaciones de exámenes, funciones que hasta entonces eran responsabilidad de los médicos y 
otros profesionales, los cuales casi nunca impartían en su totalidad la unidad de aprendizaje. Lo anterior hace evidente uno de los principales desafíos que las enfermeras debieron enfrentar, tanto por las características del aspecto señalado, como las circunstancias que en ese momento se imponía, en ese sentido Roy ${ }^{14}$ plantea que:

Las personas, vistas como sistemas adaptativos holísticos, se encuentran en continua interacción con un ambiente cambiante. Los estímulos ambientales, al entrar en contacto con un sistema, activan los subsistemas de afrontamiento y desencadenan una serie de respuestas observables a través de los modos de adaptación, las cuales buscan afrontar la situación y, de esta manera, promover la adaptación.

Ya con un ambiente de confianza y seguridad al tener la titularidad de las asignaturas, se adquieren conductas que les permitieron generar y tomar decisiones sobre la práctica, optando por reforzar los conocimientos y programación del plan de estudios, lo que implicó el conocimiento, actualización, capacidad y valoración de la responsabilidad; siendo estos los ejes fundamentales que facultaron a las enfermeras para insertarse como docentes titulares, demostrando y contribuyendo una base de aptitudes aplicables al desempeño adecuado de la disciplina, lo que direccionó a las enfermeras para lograr el objetivo de la autonomía en las asignaturas tanto prácticas como teóricas.

Un mecanismo representativo que contribuyó en la transición dentro de las instituciones de educación de enfermería, fueron los cursos de actualización, la inducción, capacitación y desarrollo del personal docente, de manera que el realizarse cursos, generaron una nueva imagen profesional, estos cursos reflejaban necesidad de superación en las instituciones de enseñanza y formación del personal ${ }^{15}$. En la década estudiada (1970-1980) los cursos de enfermería tendieron a buscar el desarrollo integral de las docentes y posteriormente del alumnado y obtener un nivel académico cada vez más alto, como se revela en la respuesta siguiente:

\section{Quisimos for talecer nuestra planta docente a través de cursos, mandamos a especializar a colegas eso abrió las puertas a las enfermeras, para poder impartir clases, para poder trabajar, para poderse especializar, para tener una mayor preparación académica (E-1).}

El desarrollo de la enseñanza forma parte de una de las funciones importantes de la docencia, que debe desempeñar la enfermera en cualquier ámbito de acción y es una actividad importante en la gerencia, donde la capacitación para el trabajo es parte complementaria de la educación, como lo menciona Balderas ${ }^{16}$, quien señala que la educación continua o enseñanza continuada, tiene como propósito reforzar la confianza del trabajador en su organización, sensibilizar a los cambios, producir innovaciones y apoyar la creatividad. La actualización docente ayudo a que las profesoras enfermeras se adaptaran al cambio de la nueva gerencia. Como lo establece Roy ${ }^{3: 1}$ al decir que el objetivo de la enfermera consiste en ayudar a las personas a adaptarse a los cambios que se producen en su auto-concepto, desempeño de roles y en sus relaciones interdependientes. Por lo que al existir una enseñanza y capacitación del personal se posibilitó el desarrollo para mejorar el desempeño, siendo indispensable que las enfermeras al ser miembros de una profesión vanguardista de ese momento, se actualizara en todas las áreas del conocimiento acerca de sus necesidades de enseñanza, ya que su ambiente de trabajo lo requería, las directoras enfermeras debieron valorar los comportamientos de las personas y los factores que influyeran en sus habilidades para la adaptación, así como impulsar los cuidados de enfermería que promueven la expansión de las habilidades adaptativas y contribuyen a mejorar las interacciones con el ambiente, mencionando Roy:2 que el entorno son las condiciones, circunstancias e influencias que afectan el desarrollo y el comportamiento de los seres humanos como sistemas adaptativos, con particular consideración de la persona y los recursos de la tierra.

Otro aspecto importante es la responsabilidad puesto que se percibe como un factor debido a que el desarrollo de la profesión y su impacto en el usuario de los servicios de enfermería, tuvo que ver con múltiples factores externos e internos propiciados por las exigencias de un nuevo orden y sistema de conducción de la educación, por ende; las universidades, sus escuelas y facultades de enfermería debieron asumir retos importantes en la formación de enfermeras, al tiempo que las nuevas dirigentes se ven obligadas a buscar mecanismos de afrontamiento, que les permitan estar actualizadas en todos los aspectos que la exigencia del momento en educación les imponía. Al indagar sobre como asumieron el compromiso frente a la dirección de las escuelas se encontró:

Con la participación de todo el profesorado invitamos a las personas de las instituciones de salud de ese momento (...) quisimos incorporar a las dirigente de enfermería que también estaban en las instituciones de salud aparte del profesorado y aparte del reconocimiento de otras instituciones, pues con hermanad (E-1)

En ese sentido, cabe decir que es notoria la visión que tienen y expresan en base a la experiencia profesional y que así mismo, tomaron, asumieron y adaptaron a su rol como dirigentes de las escuelas de enfermería, bajo este contexto, las enfermeras deben definir la función primordial que les corresponde en el logro de las metas de la organización para mostrar con resultados sus contribuciones en la profesión, como 
lo menciona Roy ${ }^{3: 3}$ la función del rol es uno de los modos existentes que atiende a los roles que asume la persona en la sociedad e implica conductas de razón de la posición de la persona, donde depende de cómo la persona interactúa con otras en una situación concreta. Por lo que en definitiva, las profesoras que adquirieron el cargo directivo de las escuelas de enfermería, debieron considerar que la función que tomaron y ejercieron se desempeño individualmente, pero siempre con apoyo de un equipo, asumiendo el rol que les correspondía para asumir la gerencia de las escuelas.

\section{Mecanismos de afrontamiento generados como gerentes}

El saber fundamenta mecanismos de afrontamiento el cual es útil para todo gerente de enfermería, para desempeñar sus funciones y administrar las acciones. Balderas menciona que esto tiene vínculo estrecho con la palabra dirigir, que consiste en guiar, orientar, conducir, encaminar, llevar al logro de objetivos, de ahí que dirigir implica tomar decisiones, mando, liderazgo ${ }^{16: 1}$. En relación al liderazgo y el cómo asumir un rol como unidad de funcionamiento en sociedad fue que se cuestiono a las entrevistadas al respecto, obteniendo las respuestas siguientes:

Demostramos capacidad de gestión y liderazgo (...) porque pudieron seguir siendo directores los médicos. Ese momento fue exitoso, nos sentíamos a gusto, nos sentíamos satisfechas (...) exigimos nuestros derechos, fui la base en esa gestión que tuvo como logro el que desarrolláramos habilidades en demostrar el liderazgo que como enfermera se necesita ( $E$-2)

Lo manifestado por las entrevistadas, refleja la importancia del puesto de dirigente y como se adaptan al rol ya que los lideres gerentes han de ser asertivos, con el grupo que dirigen, poseer iniciativa, creatividad, madurez emocional, habilidades comunicativas, ser persuasivos, observadores, accesibles, amistosos, etc., tales características permiten asumir un rol terciario, por lo que ser dirigente de una institución educativa y emprender un afrontamiento por tener un propio campo de acción, en el que debe tomar decisiones y buscar soluciones de un grupo, es de vital importancia y de gran compromiso, esto refleja la magnitud de responsabilidad en las funciones de direccionar una institución y lograr el cumplimiento de metas, siendo precursor de cambios innovadores que fortalezcan los equipos de trabajo e impacten académicamente al grado de una autonomía profesional.

Después de analizar el pasado y situarnos en el presente, las directoras participantes en la investigación aportaron información relevante al mencionar que para favorecer el estatus de las enfermeras y de la profesión en general desde el ámbito académico, existen algunas consideraciones que direccionan para alcanzar un mayor nivel tanto económico, social y sobre todo profesional, otras recomiendan la unión gremial y el incremento de enfermeras con nivel superior, como se refleja a continuación :

Necesitamos desarrollar habilidades de liderazgo, tener conocimientos fundamentados, continuar prepararnos más académicamente, conocer ampliamente lo que es nuestra profesión y el camino que queremos seguir, así como crear los cuerpos académicos, fortalecerlos y una cosa muy importante nunca perder la característica de enfermería, humanitaria en todo el sentido en todo lo que implica esa palabra para poder entender las necesidades de los compañeros y de los estudiantes. $(E-2)$

Se debe establecer una igualdad de condiciones y eso en primer lugar, es preparándote y en segundo lugar, pero no el menos importante, uniéndote con tu gremio, por que ser muy unidas nos podríamos defender, ya que ellos son muy unidos [los médicos], se defienden (E-5)

Con base en las entrevistas podemos identificar cual es la esencia de la dirigente en enfermería, es evidente que la profesión esta en transformación, pero también es cierto que la formación académica ha evolucionado desde la incursión de enfermeras a la dirección dentro de las escuelas en relación a los cambios ocurridos académicamente en los estudiantes, ya que la preparación se ha ampliado y conjuntamente fundamentado por los conocimientos de sus docentes, comparativamente cuando eran dirigidas por otros profesionistas del área de la salud, es así que, enfermería se ha incorporado a las universidades, creando programas académicos que se han enfocado hacia una mejora en la formación de profesionales e impregnar su hacer científico perfilándolo con valores éticos y morales, garantizando a la sociedad una excepcional calidad de servicios y en específico, una atención humana brindada por enfermería.

Cabe señalar que la investigación tuvo algunas limitaciones siendo las más importantes la ubicación geográfica de los sujetos de estudio, ya que se encontraban en estados distantes del centro del país por estar gozando jubilación, otra fue que las escuelas aun siendo autónomas en su estructura organizacional, no se visualiza en su organigrama la figura de directora de escuela o facultad.

\section{CONCLUSIONES}

A partir de estos resultados se puede identificar que uno de los esfuerzos cognitivos de las directoras enfermeras para ejercer funciones acordes a la reforma educativa, requerían una orientación especial así como un nivel relativamente alto 
de educación con el fin de desarrollar conocimientos y aptitudes en un sentido más amplio para adaptarse a las necesidades requeridas y ejercer el rol como dirigente de manera eficaz.

Otro aspecto identificado fueron los estímulos que intervienen en el proceso de adaptación de las dirigentes de las escuelas de enfermería, como son: la necesidad del proceso académico que requería de dirección y orden para desarrollar un plan de estudios sólido con una visión dentro de la disciplina, así mismo, se identificó que en ese proceso de transición, las dirigentes afrontaron retos; los cuales les exigían responsabilidad para la creación de programas acordes a la profesión, estos fueron reestructurados y evaluados y al obtener independencia se pudieron establecer dichos programas educativos con base a las necesidades de la población. De igual manera, fueron atendidas las exigencias de capacitación del personal docente, creando diferentes niveles académicos y especialización para la impartición de las unidades de aprendizaje, esto permitió que las enfermeras docentes obtuvieran la titularidad de las materias, basadas en las aptitudes y el conocimiento de cada una de ellas.

Uno de los esfuerzos comportamentales de las dirigentes fue que al obtener autonomía gerencial, debieron dar ejemplo de superación, deduciéndo con esto que, las directoras en esa fase de cambio lograron sus afrontamientos, contribuyeron a generar los modos de adaptación positivos para alcanzar un modelo innovador de dirigentes, indudablemente al tener conocimientos, preparación y propósitos personales e institucionales, les favoreció alcanzar la adaptación.

La transformación que tuvo la enfermería al pasar de una práctica dependiente del área médica, a una disciplina autónoma desde el punto de vista académico, permitió ofrecer una formación igualitaria al de otras profesiones, reflejando con esto un crecimiento profesional y participativo de las enfermeras.

De esta manera se tiene un punto de partida para explicar y comprender el nivel de formación particular en esa temporalidad, debido que al ser dependientes de la medicina inhibía el crecimiento profesional de las enfermeras, sin embargo se puede visualizar que uno de los esfuerzos cognitivos, es reflejado en el grado de competencia de las dirigentes de enfermería, ya que direccionó el sustento del desarrollo educativo profesional actual.

\section{REFERENCIAS}

1.Robles M. Educación y sociedad en la historia de México. $3^{\mathrm{a}}$ ed. México: Siglo Veintiuno; 1979. 242 p.

2.Moreno FME, Alvarado GAM. Aplicación del modelo de adaptación de Callista Roy en Latinoamérica: revisión de la literatura. Aquichan. 2009 abr; 9(1): 62- 72
3.Marriner TA, Raile AM. Modelos y teorías en enfermería.7ª ed. Madrid: Elsevier Mosby; 2011.

4. Barbato RG, Corre AAK, Souza MCBM. Aprender em grupo: experiência de estudantes de enfermagem e implicações para a formação profissional. Esc Anna Nery. 2010 jan/mar; 14(1): 48-55

5.Roy $C$. Relating nursing theory to nursing education: a new era. Nurse Educ Prat. 1979; 2(1): 55-68.

6.Análisis de los conceptos del modelo de adaptación de Callista Roy. Aquichan [on- line] 2002; [citado 2010 ago 2]; [aprox. 8 telas] Disponible en: http://redalyc.uaemex.mx/redalyc/src/inicio/ ArtPdfRed.jsp?iCve=74120204\#

7. Roy C, Chayaput P. Coping and adaptation processing scale english and thai versión. Roy Adaptation Association Review. 2004; 6(2): 4 -6.

8.Andrews H, Roy C. Essentials of the Roy Adaptation Model. Norwalk, CT: Appleton Century Crofts.

9.Whetsell M., Frederickson K., Agulera P., Maya JL. Nivel de bienestar espiritual y de fortaleza relacionados con la salud em adultos mayores. Aquichan. 2005; 5(1): 74

10.Roy C, Andrews H. The Roy Adaptation Model. Stanford: Appleton y Large; 1999.

11.Cárdenas BL. La profesionalización de enfermería en México. Un análisis desde la sociología de las profesiones. Barcelona: Pomares; 2005.

12.Merchán N, Rodríguez-AC, M.Barca, J.Carrero A, Galindo A, Rojo R. La evolución de los contenidos teórico prácticos en enfermería médicoquirúrgica en las tres últimas décadas. [on-line]; [citado 2011 febr 20]; Disponible en: http://www.uclm.es/ab/enfermeria/revista/numero\%206/ evolconteoprac6.htm

13.Perfil profesional de enfermería técnica y licenciatura: similitudes y diferencias. Dozal RM, Padilla LM, FEMAFEE2004. [on- line] ; [citado 2010 ago 25] ; Disponible en: http://femafee.net/archivo/modelo.pdf.

14.Gutiérrez AMC, et al. Adaptación y cuidado en el ser humano una visión de enfermería. Bogotá: Universidad de la Sabana Colombia; 2007.

15.Barrigüete GLM., Penna TM. Organización y gestión de instituciones y programas educativos. Madrid: Catarata; 2011.

16.Balderas PML. Administración de los servicios de enfermería, $5^{a}$ ed. México (DF): Mc Graw- Hill; 2009. 